\title{
Neues Modell zur Finanzierung der ärztlichen Weiterbildung im Allgemeinen und unter DRG
}

\author{
Die Finanzierung der ärztlichen Weiterbildung ist ein Thema, das Politik, Spital- und \\ Universitätsleitungen sowie das Schweizerische Institut für ärztliche Weiter- und \\ Fortbildung (SIWF) bzw. dessen Vorgängerorganisation seit über zehn Jahren be- \\ schäftigt. Sehr unterschiedlich sind die Meinungen bezüglich der Institutionen oder \\ Personen, welchen die Finanzierung überantwortet werden könnte. Eine Studie im \\ Auftrag des SIWF stellt eine Poollösung zur Diskussion.
}

Max Giger ${ }^{a}$, Harry Telser ${ }^{b}$

a Dr. med., Präsident SIWF, c/o FMH, 3000 Bern 15

b Dr. oec. publ., Polynomics AG, Olten

\begin{abstract}
Die Finanzierung der ärztlichen Weiterbildung muss im Lichte des Bedarfs an ärztlichen Leistungen bzw. der künftigen ärztlichen Arbeitskraft (Workforce) betrachtet werden. Seit über 20 Jahren genügt die Anzahl der in der Schweiz weitergebildeten Ärztinnen und Ärzte nicht, um den Bedarf in den Institutionen zu decken. Heute stammen $44 \%$ der Ärztinnen und Ärzte, welche eine Weiterbildung zu einem der 43 schweizerischen Facharzttitel absolvieren, aus dem Ausland. Die Schweiz ist weiterhin auf die ausländischen Assistenzärztinnen und Assistenzärzte angewiesen. Es dauert mindestens sechs Jahre, bis die von den medizinischen Fakultäten eingeleiteten Massnahmen zur Steigerung der Aufnahmekapazität um 15-20\% sich in den Spitälern auswirken. So muss die Schweizer Weiterbildung weiterhin attraktiv bleiben. Die Attraktivität basiert auf den folgenden drei Elementen: Gutes Arbeitsklima in den Schweizer Spitälern, gute supervidierte Vermittlung der Weiterbildungsinhalte, hohe Lebensqualität. Zu letzterer trägt auch die Entlöhnung während dieser wichtigen Phase der beruflichen Entwicklung bei. Diese steht in direktem Zusammenhang mit der Finanzierung der Weiterbildung.
\end{abstract}

\section{«Durch die Sicherung der Finanzierung der Weiter- bildung kann auch deren Effizienz und Attraktivität beeinflusst werden»}

\section{Über 800 neue Weiterbildungsstellen in 4 Jahren}

Durch die Sicherung der Finanzierung der Weiterbildung kann auch deren Effizienz und Attraktivität beeinflusst werden. Besonders die Effizienzsteigerung liegt im Interesse der Assistenzärztinnen und Assistenzärzte, welche oft die für die Erteilung des Facharzttitels notwendigen Untersuchungen und Eingriffe nicht in der optimalen Zeitspanne, das heisst inner- halb von fünf bzw. sechs Jahren, durchführen können. Zur selbständigen Berufsausübung wird vom Medizinalberufegesetz (MedBG) eine minimale Weiterbildungsdauer von drei Jahren vorgeschrieben. Deshalb bilden sich die meisten diplomierten Ärztinnen und Ärzte weiter. Die Weiterbildung erfolgt am und mit dem Patienten und schliesst je nach Weiterbildungsprogramm zwischen zwei und sechs Stunden theoretische Veranstaltungen wöchentlich ein. Im Jahr 2009 sind über 9000 Weiterbildungsstellen belegt, und zwar $3 \%$ im Tessin, 30\% in der Romandie und $67 \%$ in der Deutschschweiz. Bis auf wenige Ausnahmen befinden sich die Weiterbildungsstellen an den rund 1200 öffentlichen und öffentlich-rechtlichen Spitälern und Institutionen. Diese stehen grossmehrheitlich für die Weiterbildung ein, einerseits im Interesse der Leistungserbringung und andererseits als lernende Organisationen im Interesse der Innovation und Sicherung der künftigen Workforce. Zusätzlich findet die Weiterbildung in der ambulanten Medizin, namentlich der Hausarztmedizin, in rund 650 Arztpraxen statt.

Die Unterstellung der Assistenzärztinnen und -ärzte unter das Arbeitsgesetz führte dazu, dass ein Teil der Weiterbildungskosten, den bis anhin die Assistenzärzte (durch höhere Arbeitszeiten) getragen hatten, neu von den Spitälern finanziert werden muss. Kurzfristig resultierte dies darin, dass in den letzten vier Jahren über 800 neue Stellen geschaffen wurden. Dies entspricht einem Weiterbildungsjahrgang. Langfristig reduziert diese Umverteilung der Weiterbildungskosten zu den Spitälern jedoch deren Anreize, ärztliche Weiterbildung anzubieten. Viele Institutionen haben bereits begonnen, die Investition in die ärztliche Weiterbildung zurückzufahren.

\section{Verschärfung der Finanzierungsprobleme durch DRG}

Die generelle Einführung von Fallkostenpauschalen (DRG) wird dieses Problem noch verstärken. Die Kantone werden zwar verpflichtet sein, gemeinwirtschaftliche Leistungen, zu denen die Weiterbildung gemäss 
aktueller Gesetzeslage zählt, abzugelten. Ob diese Beiträge aber die Kosten der ärztlichen Weiterbildung in den Spitälern decken werden, ist unklar, weil die Kosten unbekannt und nur sehr schwer und ungenau zu ermitteln sind. Da ärztliche Weiterbildung in den Spitälern und in der Ambulanz in Kuppelproduktion mit Gesundheitsleistungen, Forschung und Lehre angeboten wird, lassen sich die Kosten kaum isolieren. Grobe Abschätzungen kommen zu Werten zwischen 17000 Franken bis hin zu 168000 Franken pro Assistenzarzt und Jahr. Das Auseinanderklaffen dieser Werte beruht unter anderem darauf, dass unterschiedliche Methoden zum Einsatz kamen, die von Einzelanalysen in einem Spital bis zu ökonometrischen Auswertungen der gesamtschweizerischen Krankenhausstatistik reichten. Die ärztliche Weiterbildung wird im Zuge der Umstellung der Spitalfinanzierung dementsprechend vor grosse Herausforderungen gestellt.

\section{Vorschlag: Einrichtung eines Weiterbildungs-Pools}

Aus einem neu zu errichtenden Fonds, der - in Anlehnung an die aktuelle Situation der dualen Spitalfinanzierung - primär durch die Kantone zu finanzieren wäre, soll denjenigen Spitälern und Institutionen, welche effiziente Weiterbildung anbieten, zu Beginn pro besetzte Stelle ein Betrag in der Grössenordnung von 17000 Franken - dem tiefsten Wert der bekannten groben Kostenabschätzungen - ausgerichtet werden. Die Weiterbildung in Arztpraxen könnte auch nach diesem Modell unterstützt werden. Der Pool kann unter Aufsicht der Kantone und des Bundes durch das SIWF verwaltet werden.

Ein grosser Vorteil einer solchen Poollösung liegt darin, dass mit den ausgerichteten Beträgen an die Spitäler steuernd ins Weiterbildungssystem eingegriffen werden kann. Ohne Kenntnis über die Kosten der ärzt-

\section{«Ein grosser Vorteil einer solchen Poollösung liegt darin, dass mit den ausgerichteten Beträgen an die Spitäler steuernd ins Weiterbildungssystem eingegriffen werden kann»}

Die ärztliche Workforce muss jedoch aus Gründen der Versorgungssicherheit der Bevölkerung unabhängig von der Abgeltung der Leistungen der Spitäler und Institutionen gewährleistet werden. Deshalb muss die Finanzierung der Weiterbildung gesichert werden, und zwar sowohl im stationären als auch im ambulanten Bereich. Im Auftrag des SIWF erstellten Harry Telser und Karolin Leukert von Polynomics eine Studie zur Finanzierung der ärztlichen Weiterbildung aus ökonomischer Sicht (Vollversion unter www.polynomics.ch oder www.siwf.ch). Sie stellen nach Aufzeigen allgemeiner Überlegungen zur Weiterbildung und exemplarischer Finanzierungsmodelle (u.a. Deutschland, USA, Neuseeland) für die Schweiz eine Poollösung zur Diskussion. lichen Weiterbildung haben zu müssen, können die Beiträge erhöht oder gesenkt werden, was den Spitälern Anreize gibt, die Zahl der Weiterbildungsplätze zu steigern oder zu verringern. Zusätzlich kann mit differenzierten Beträgen nach Fachgebiet Einfluss auf die Verteilung der Facharztdisziplinen genommen werden. Die Finanzierung der effizienten Weiterbildung kann so gesichert werden. Der Ball ist damit bei den Verantwortlichen für die Gesundheitsversorgung, den Direktionen der kantonalen Gesundheitsdepartemente, welche sich mit der vorgeschlagenen Poolösung noch in diesem Herbst vertieft auseinandersetzen werden. 